\title{
Substituent Effects of Amines on Genipin Dye Formation - Insights into Dye Origins
}

\author{
Fiona Jeeva and Christopher B. Caputo*
}

Abstract: Genipin, a naturally occurring iridoid, represents an interesting class of reactive dyes. This colorless molecule produces brilliant blue dyes in the presence of primary aliphatic amines. The mechanism behind this unique reaction has been suggested to proceed via an oxidative polymerization reaction, albeit few studies have investigated this process. By utilizing aromatic amines, we demonstrate that the dye absorption properties can be redshifted to form green dyes as opposed to the blue that is most associated with aliphatic amines. Characterization of the green material with gel-permeation chromatography, mass spectrometry, and electrochemical experiments suggest an alternate structure of the dye molecules and led us to revisit the mechanism behind this reaction. Generation of dyes from the reaction of genipin and methylamine or various aniline derivatives revealed the complete absence of polymeric material and instead revealed a material that contains an open-shell configuration as determined by EPR spectroscopy. Herein we report the first findings that dyes formed from the reaction of genipin with amines form persistent radicals that are likely responsible for their vibrant colors, as opposed to the previously suggested polymer formation.

Introduction: Synthetic dyes are ubiquitously used for applications as diverse as inks, textiles, and food colorants thanks to their high stability and low cost. ${ }^{[1]}$ Despite this, research into renewable dyes has been growing due to a large paradigm shift in environmental awareness. Common naturally-occurring dyes include annatto, saffron or indigo, but drawbacks exist to their synthetic analogues, including stability, brilliance and cost. ${ }^{[2]}$ One lesser-studied candidate for application as a dye is genipin, a naturally occurring iridoid derived from the extract of the fruit Gardenia Jasminoides Ellis. Genipin is a white solid, but it will readily form a dark blue dye upon 
reactions with primary amines in the presence of oxygen gas. These blue dyes are safe to eat, and are often used as blue food colourants; ${ }^{[3,4]}$ in fact, our group has recently used this to develop edible genipin-based sensors for biogenic amine detection and food spoilage ${ }^{[5]}$. Another attractive feature of genipin dyes is that they are also more robust in terms of temperature and light sensitivity than Indigo Blue - another natural blue dye that is commonly employed in the textile industry. ${ }^{[3]}$ Genipin has found numerous application in diverse fields, most commonly used as a non-toxic cross-linking reagent for biopolymers and materials. ${ }^{[6-10]}$ Furthermore, it has found applications as a fingerprint reagent, ${ }^{[11,12]}$ as well as in various medical applications which exploit its cross-linking properties. ${ }^{[13,14]}$

The mechanism behind genipin dye formation has been less studied compared to its applications, however the reaction has been proposed to proceed via an initial water-facilitated ring-opening of the hemiacetal, forming a dialdehyde intermediate $\mathbf{1}$ (Scheme 1, a ${ }^{[15]}$. This tautomer can readily undergo two consecutive Schiff-base condensation reactions with a primary amine yielding proposed intermediate 2 . This molecule is not redox stable and has been proposed to undergo a redox self-catalytic cycle to eliminate water. ${ }^{[16]}$ This step is rather uncontrolled and results in the formation of a complex mixture of genipin-derived monomers, dimers, trimers, and tetramers illustrated as in Scheme 1b. Some of these products were isolated and elucidated by Touyama and co-workers (see Scheme 1, b). ${ }^{[17,18]}$ These results help explain the well-observed crosslinking behavior of genipin. This mixture of products has been reported to result in a red/brown pigment when isolated under an inert argon atmosphere. However, exposure to air results in the rapid formation of a brilliant blue dye. Touyama and co-workers propose that an oxygen-induced radical polymerization event occurs to form materials composed of 40-44 units, with an average MW of $8970 \mathrm{~g} / \mathrm{mol}^{[6,17]}$ 
a)

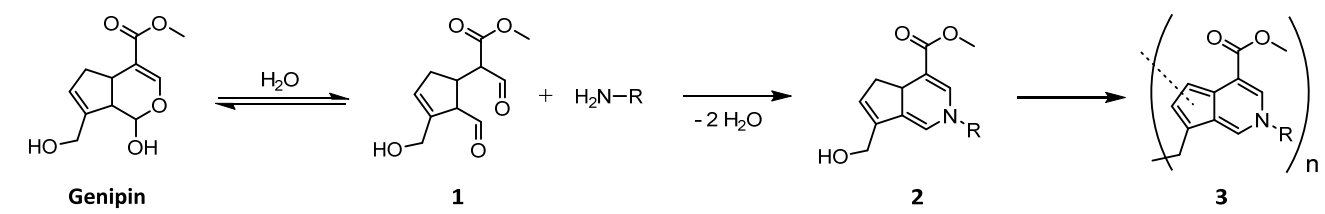

b)
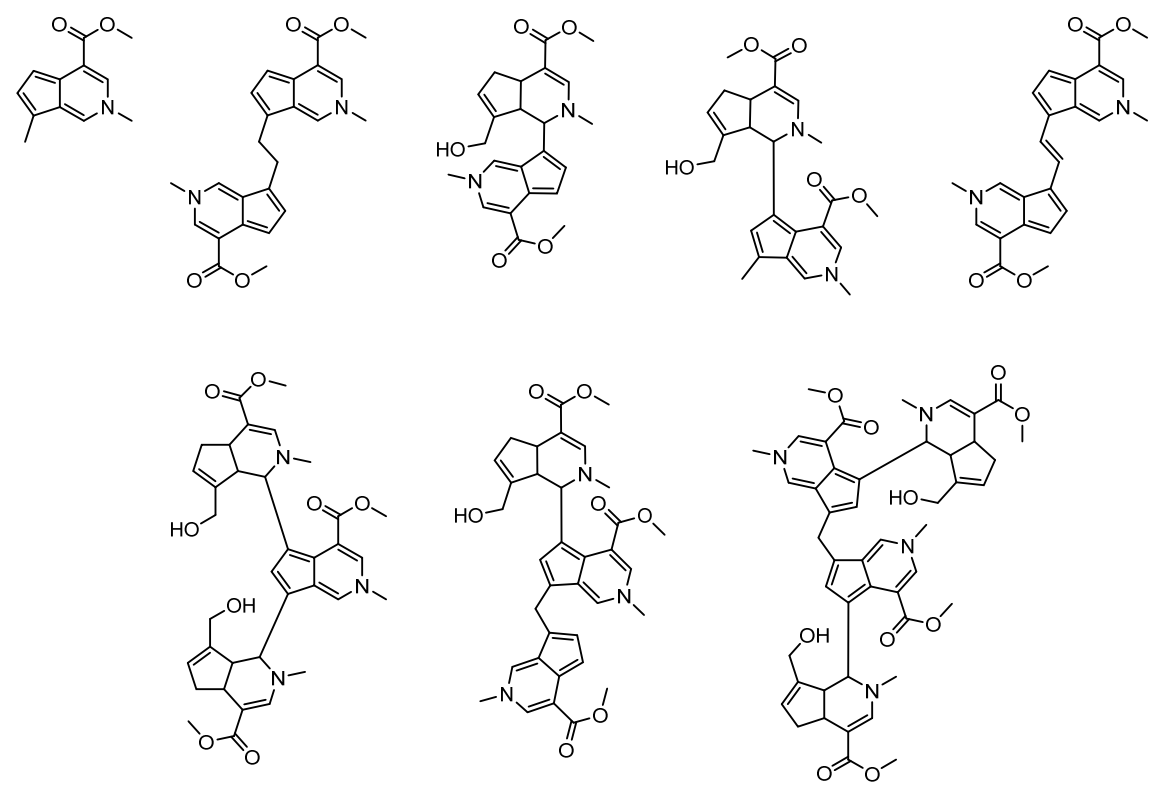

Scheme 1. a) Genipin ring opening and reaction with amines b) Genipin-methylamine products characterized by Touyama.

This mechanism has been generally accepted by the community, however despite the broad applications that genipin has found, the mechanism has yet to be fully understood and several questions remain. Further, the generation of these blue "polymeric" dyes has only been studied using methylamine or various primary amino acids. This presents one glaring omission in the research field, which is the effect that the amines themselves have on the color of the dye. Naturally, we set out to investigate what impact the amine structure had on the resulting dye color. We hypothesized that using aromatic amines — as opposed to aliphatic amines — will extend the $\pi$-conjugation of the system and therefore cause a bathochromic shift in the absorption. Unexpectedly, this study allowed us to further illuminate insights into the structure of the dyes, 
suggesting that a polymerization event does not occur; instead a stable open-shell configuration appears to be central in the resulting coloration.

\section{Results and Discussion}

Genipin Reactivity with Aromatic Amines: Genipin dyes derived using primary amines or amino acids typically show a characteristic absorption band with a maximum at $590 \mathrm{~nm}$, with slight variation depending on the amine chosen, corresponding to what was previously presumed to be the blue polymer. ${ }^{[19]}$ We began our studies by exploring the reactivity of genipin with the simplest of aromatic amines — aniline. An equimolar mixture of genipin and aniline was dissolved in 10 $\mathrm{mL}$ of methanol and stirred at room temperature for 48 hours. To our delight, the reaction proceeded in a similar fashion, first through a reddish-brown intermediate, but unlike in reactions with aliphatic amines, the reaction resulted in a green dye as opposed to a blue material $\left(5, \lambda_{\max }=\right.$ $630 \mathrm{~nm}$ ). This resulting bathochromic shift in emission lent credence to our original hypothesis. However, aniline is known to undergo oxidative polymerization to produce polyaniline (PANI) emeraldine salt which is also green in color with $\lambda_{\max }=663 \mathrm{~nm} \cdot{ }^{[20]}$ Unfortunately, ${ }^{1} \mathrm{H}$ or ${ }^{13} \mathrm{C}$ NMR analysis does not provide any insights into the product formed, due to the complex nature of oligomers formed. Therefore, to ensure that the green pigment was in fact a product of the reaction with genipin and not simply an emeraldine salt, the reaction was carried out using $p$-toluidine, which contains a methyl group on the para-position of the aromatic ring, effectively blocking the site of polymerization. The reaction of $p$-toluidine with genipin also gave a green product $\left(\mathbf{6}, \lambda_{\max }\right.$ $=624 \mathrm{~nm}$ ), suggesting that the green color formed was exhibited by the hypothesized polymeric material.

We sought to expand the scope of aromatic amines, while studying both steric and electronic effects on dye formation. To investigate the impact that steric bulk of the aromatic ring has on the 
reaction, both 2,4,6-trimethylaniline and 2,4,6-tri(tert-butyl)aniline were investigated. In this case, the methyl-substituted aniline did react with genipin under our conditions to yield a dark green dye (7) with a $\lambda_{\max }$ feature at $621 \mathrm{~nm}$, slightly blue-shifted compared to the aniline and $p$-toluidine variants. Unsurprisingly, the reaction with the bulkier $t$-Bu analogue did not proceed, as no colour change was observed. This result supports the idea that steric bulk around the amino substituent could play a role in the ability of genipin to form dyes. In addition, fluorinated amines such as 3,4,5-trifluoroaniline and pentafluoroaniline showed no reactivity with genipin under these standard conditions, likely due to the electron deficiency of the amine. In total, the reaction was carried out with 10 different aromatic amines to understand the impacts on the absorption properties. The $\lambda_{\max }$ of the dyes with the corresponding aromatic amine can be seen in Table 1 along with the corresponding stacked UV-Vis spectra of the region of interest in Figure 1. The individual UV-Vis spectra of the dyes can be found in Figures S5-S13. Molar absorptivities were not calculated due to the uncertainty in the concentrations of the species in the complex mixture of products formed in the reaction. Thus, UV-Vis samples were analyzed at a uniform concentration of $0.025 \mathrm{mM}$ calculated from the genipin reactant, as the distribution of molecular weight products made the calculation of the concentration impossible.

To elucidate the impact that extending the $\pi$-conjugation has on the resulting dye, biphenyl, anthracene and pyrene derivatives were studied (Entry 9-12). While a bathochromic shift of the $\lambda_{\max }$ wavelength was observed with respect to the methylamine dyes, there was no discernible correlation between the size of the aromatic system and the red-shift in emission. This is demonstrated by the fact that the two aminoanthracene derivatives have a slight blue-shift in their emission maxima compared to the parent aniline dye. This result led us to question the nature of the genipin-derived dyes. Was it possible that they were not polymeric at all? 


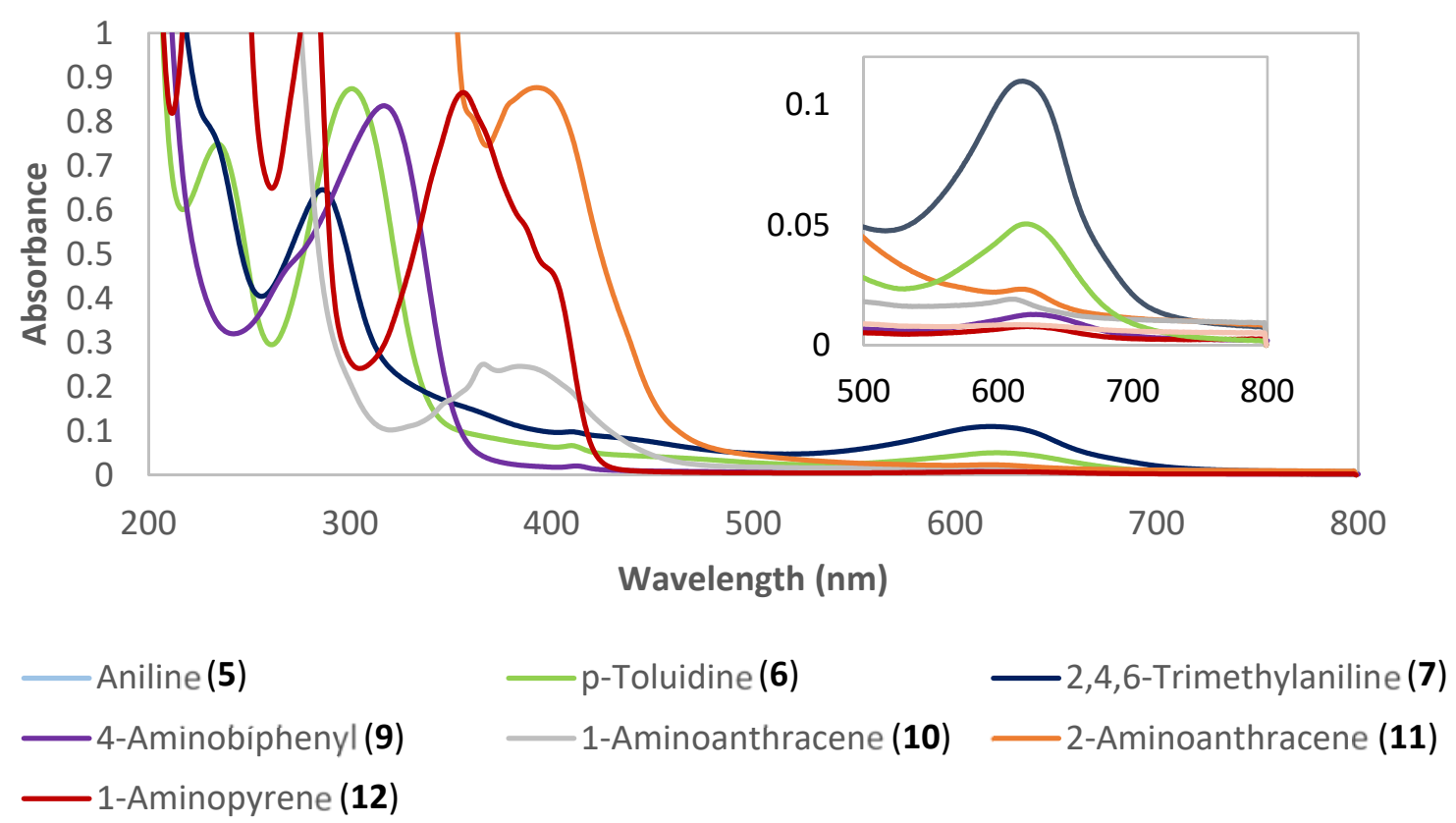

Figure 1. UV-Vis absorption spectra of genipin-amine products with $0.025 \mathrm{mM}$ Genipin concentration. Inset: UV-Vis absorption spectra from 500-700 nm.

Determination of Genipin Dye Composition: With the confirmation that genipin does react with aromatic amines, resulting in new green dyes, we set out to investigate any differences in the polymer length between the polymer formed with methylamine, and the prospective polymers formed with these aromatic amines. The product formed from the reaction of $p$-toluidine with genipin (6) was chosen as a model to compare to the methylamine material. Gel Permeation Chromatography (GPC) was performed on these samples, however, much to our surprise, no polymers were found (Figure S15). The lower limit of the GPC was $1000 \mathrm{~g} / \mathrm{mol}$, indicating that the only compounds present in the reaction mixture were small molecules; in the case of $\mathbf{6}$, the largest genipin-derived dye molecule detected under $1000 \mathrm{~g} / \mathrm{mol}$ would be that of a trimer at $\sim 831$ $\mathrm{g} / \mathrm{mol}$. In response to this, we replicated the methylamine reaction with genipin as reported by Touyama $^{[17]}(4)$, forming the dark blue material to determine if the polymers were, in fact, only formed with aliphatic amines. We analyzed this sample using MALDI-TOF spectrometry to detect 
both oligomeric and polymeric products. MALDI-TOF analysis revealed similar findings to those from GPC, where the largest compound in the reaction mixture had a $\mathrm{m} / \mathrm{z}$ of 832.001 , the approximate mass of the previously reported genipin-methylamine tetramer (Figure S17). Interestingly, this molecule was reported by Touyama ${ }^{[17]}$ as a red solid (11, Scheme 1b), and not the source of the blue dye.

Further analysis of $\mathbf{4}$ using electrospray ionization mass spectrometry (ESI-MS) revealed the presence of a variety of compounds alongside the tetramer, with $\mathrm{m} / \mathrm{z}$ values in the ranges of 200300, 400-500 and 600-700 corresponding to monomers, dimers, and trimers, respectively (Figure S19). Of the notable peaks, $\mathrm{m} / \mathrm{z}$ values of 220.10 and 242.08 correspond to the $[\mathrm{M}+\mathrm{H}]$ and $[\mathrm{M}+\mathrm{Na}]$ peaks of the monomer of $\mathbf{4}$ in Figure 2a. The commonly proposed dimer $\mathbf{4}$ also appears to be present with a $[\mathrm{M}+\mathrm{H}]$ peak at $403.16 \mathrm{~m} / \mathrm{z}$, and the $[\mathrm{M}+\mathrm{H}]$ of trimer 4 at $624.27 \mathrm{~m} / \mathrm{z}$. ESI-MS of 6 showed very similar distribution of products, with $\mathrm{m} / \mathrm{z}$ ranges of 250-400, 550-700 and 800-900, suggesting the formation of monomers, dimers, and trimers as well (Figure S20). Some postulated products can be seen in Figure $2 \mathbf{b}$, with peaks at $298.14 \mathrm{~m} / \mathrm{z}, 555.23 \mathrm{~m} / \mathrm{z}$ and $832.33 \mathrm{~m} / \mathrm{z}$, corresponding to the $[\mathrm{M}+\mathrm{H}]$ peaks of a monomer, dimer, and trimer of $\mathbf{6}$, respectively. However, the monomer in this case appeared to be the most abundant and no tetramer was observed, as anticipated by GPC. It should be noted that due to the complex mixture of products in the reaction mixture, the proposed structures are only a few of a vast assortment of molecules. Thus, despite the large variety of molecules seen in the mass spectrometry, the molecular weights are grouped in what is consistent with monomers, dimers, and trimers. Consequently, we were surprised to find that we were able to obtain the highly colored dyes, despite the absence of polymers. This led us to revisit the question — what makes genipin-derived dyes so brilliantly colored? 
a)

b)

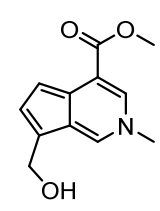

$\mathrm{m} / \mathrm{z}: 219.0895$

4 - Monomer
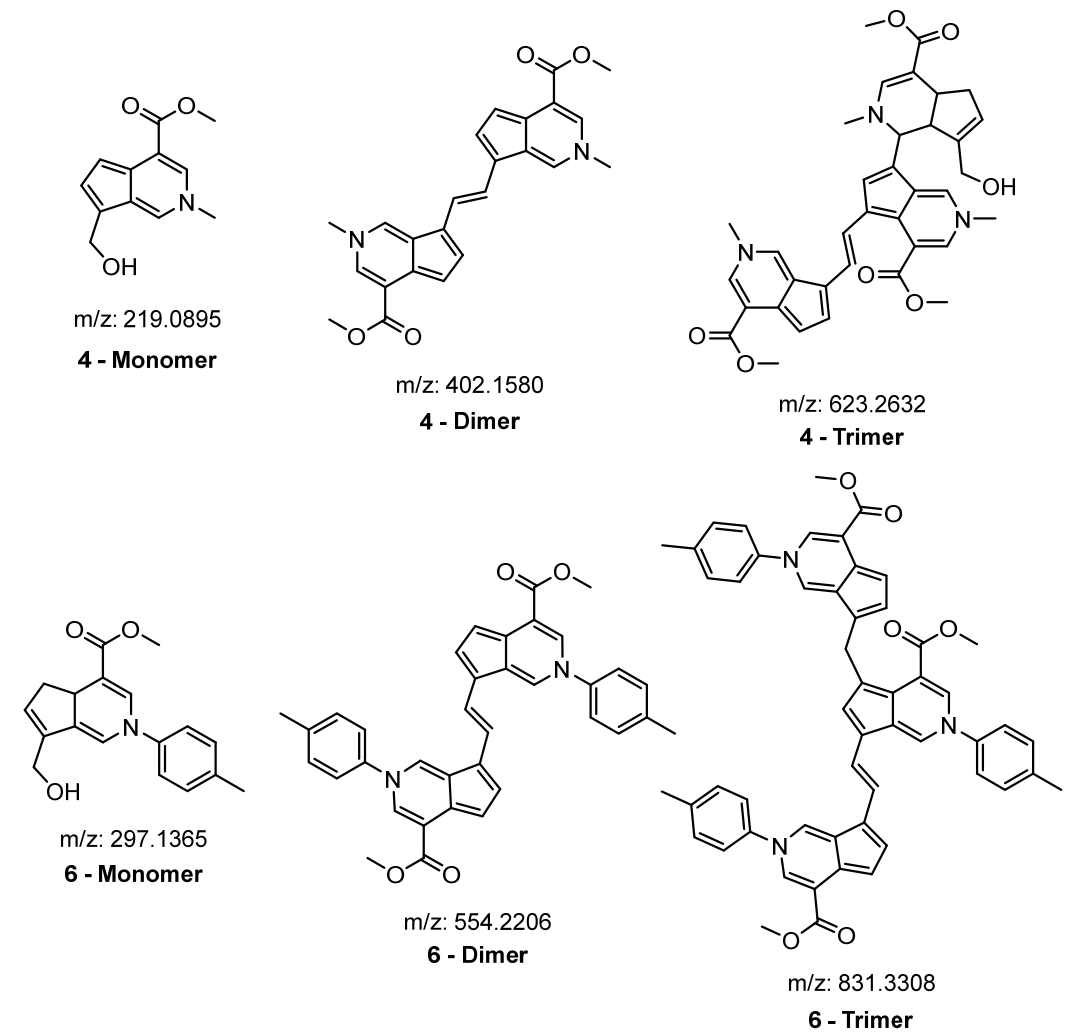

Figure 2. Proposed structures of compounds formed found in the a) genipin-methylamine dye (4) and b) genipin-p-toluidine dye (6) as indicated from mass spectrometry.

Revisiting the Origins of Genipin-Derived Dyes: With the assumption that the blue dye of 4 was a result of an oxidative polymerization reaction, we sought to study this product by ${ }^{1} \mathrm{H}$ and ${ }^{13} \mathrm{C}$ NMR spectroscopy to gain insight into the structure. However, despite a sufficiently concentrated solution, no resonances aside from the solvent peaks were observed in both the ${ }^{1} \mathrm{H}$ or ${ }^{13} \mathrm{C}$ NMR spectra (Figure S1-S2). The absence of polymers in the dark blue dye, accompanied by the silence in the NMR spectra led us to suspect the presence of an unpaired electron causing the dye coloration. The NMR experiments were repeated with 6, however analysis using NMR spectroscopy is difficult on these reactions as the complex product distribution led to complicated spectra (Figure S3). Nevertheless, the green dye was purified using silica gel chromatography and the product was reanalyzed, yielding the same results as 4 (Figure S4). While it was widely 
accepted that genipin-amine products undergo an oxygen-induced radical polymerization (videsupra), to our knowledge it has yet to be suggested that any of the final compounds themselves contain an open shell configuration that is responsible for the color. Naturally, EPR spectroscopy was performed on both the dyes of $\mathbf{4}$ and $\mathbf{6}$ to investigate the presence of an unpaired electron. Both EPR spectra were obtained at room temperature, with 6 dissolved in a $\mathrm{CH}_{2} \mathrm{Cl}_{2}$ solution, while 4 was run in the solid state. The latter was due to limited solubility in solvents aside from water and alcohols (both of which tend to have higher than ideal dielectric constants for effective EPR measurements). Both 4 and 6 exhibited a broad singlet around $0.3331 \mathrm{G}$ and $0.3464 \mathrm{G}$, respectively, suggesting that there is an unpaired electron present in the dye product itself (Figure 3). Both species have a g-value of 2.002, consistent with the approximate range of a carbon or nitrogen centered radical. ${ }^{[21,22]}$ We further obtained EPR spectra for the series of aromatic amines investigated (see Table 1) and found that all had the presence of an unpaired electron with all gvalues around 2.002 (Figures S21-S29). These g-values can be found in Table 1 and all show a lack of hyperfine-coupling, which could indicate an electronic delocalization of the unpaired electron across the entire molecule. ${ }^{[23]}$ 

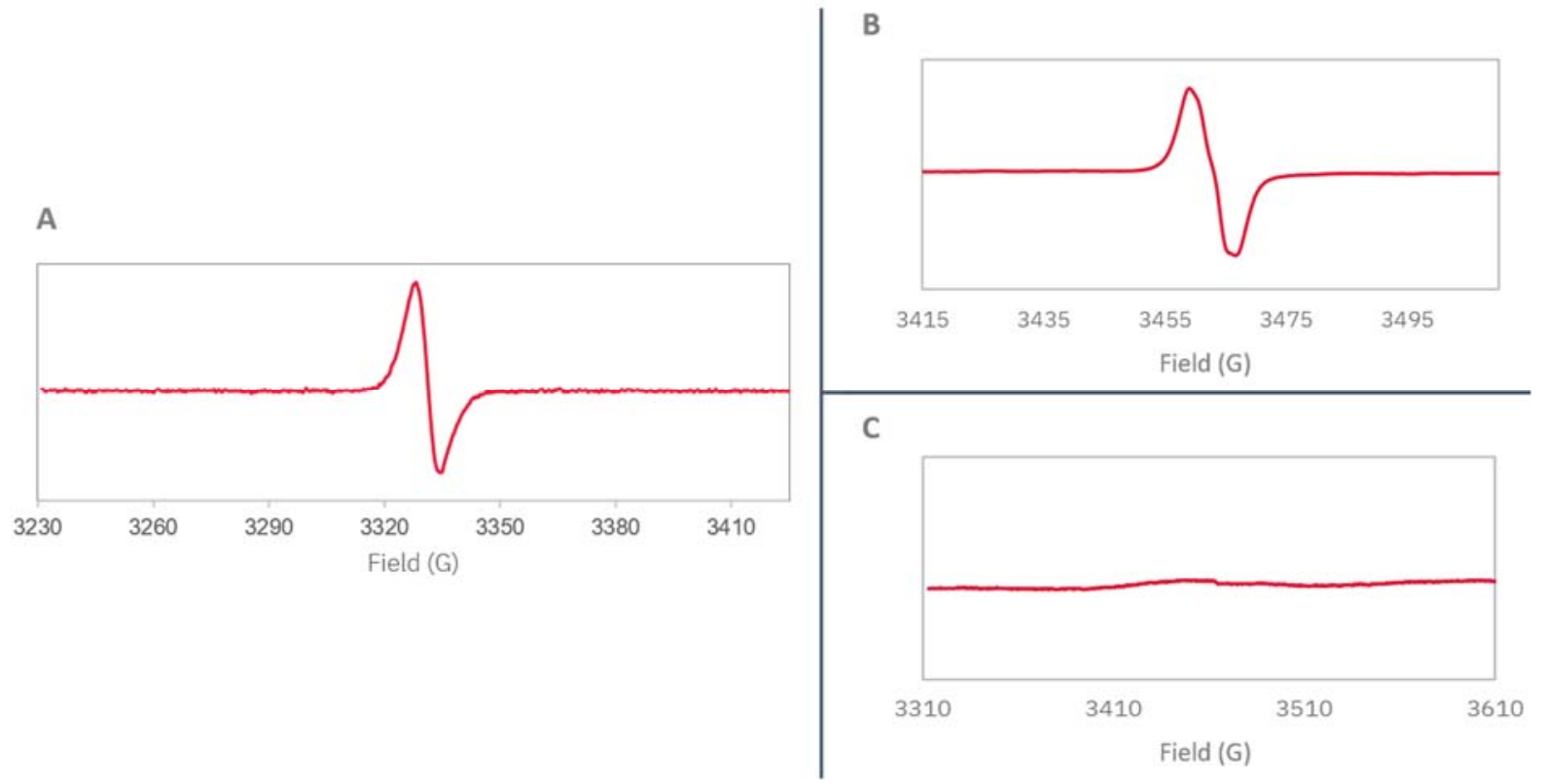

Figure 3. EPR Spectra of A) 4, B) 6 and C) unoxidized 6

Table 1. List of amine substrates, their corresponding genipin dye $\lambda_{\max }$ values and their corresponding g-values.

\begin{tabular}{llll} 
Entry & RNH 2 & $\lambda_{\max }(\mathrm{nm})$ & $\mathrm{g}$-value \\
\hline $\mathbf{4}$ & Methylamine & 590 & 2.002 \\
\hline $\mathbf{5}$ & Aniline & 630 & 2.002 \\
$\mathbf{6}$ & p-toluidine & 624 & 2.002 \\
$\mathbf{7}$ & 2,4,6-trimethylaniline & 621 & 2.002 \\
$\mathbf{8}$ & 2,4,6-tri(tertbutyl)aniline & $\mathrm{N} / \mathrm{A}$ & $\mathrm{N} / \mathrm{A}$ \\
$\mathbf{9}$ & 4-Aminobiphenyl & 629 & 2.002 \\
$\mathbf{1 0}$ & 1-Aminoanthracene & 613 & 2.002 \\
$\mathbf{1 1}$ & 2-Aminoanthracene & 619 & 2.002 \\
$\mathbf{1 2}$ & 1-Aminopyrene & 640 & 2.001 \\
$\mathbf{1 3}$ & Pentafluoroaniline & N/A & N/A \\
$\mathbf{1 4}$ & 3,4,5-trifluoroaniline & N/A & N/A \\
\hline
\end{tabular}


As a control experiment to gain further information on the impact of the radical on the color of these species, the red intermediate from the reaction of genipin and p-toluidine was prepared using Schlenk techniques to exclude the presence of oxygen gas. No EPR resonance was observed in this sample, (Figure 3c) indicated that the red intermediate dye has a closed shell configuration. Consequentially, this supports the hypothesis that the source of the blue/green colors is the presence of a stable open-shell configuration.

Finally, to further confirm that the color change from red to blue arises from this redox process, we undertook cyclic voltammetry $(\mathrm{CV})$ and spectroelectrochemical studies of these dyes. CV was run on the oxidized blue/green dyes of 4 and 6 in acetonitrile containing $0.1 \mathrm{M} \mathrm{TBAPF}_{6}$ as the electrolyte. The $\mathrm{CV}$ of $\mathbf{4}$ showed a quasi-reversible reduction potential at $-1.4 \mathrm{~V}$ and $-0.9 \mathrm{~V}$ for $\mathbf{6}$ (vs Ag/ $\mathrm{AgCl}$; Figures S30-31). This reduction peak is indicative of two possible outcomes. The first (pathway i; Figure 5c) would be that the reduced species is the same as the parent red, unoxidized product. Alternatively, reduction of the blue dye could generate a new, unknown species (pathway ii; Figure 5c).

Thus, spectroelectrochemistry was performed on methylamine- and $p$-toluidine-derived dyes to aid in the elucidation of what is occurring upon reduction. The spectroelectrochemical spectra demonstrated that approaching the reduction potentials of 4 correlated to a decrease in the absorption feature at $\lambda=593 \mathrm{~nm}$, the absorption corresponding to its characteristic dark blue colour (Figure 5a). Concurrently, there also was a decrease in the absorption feature at $\lambda=237 \mathrm{~nm}$ with a corresponding increase in intensity at $\lambda=278 \mathrm{~nm}$, with a long shoulder giving a slight absorption in the 400-500 $\mathrm{nm}$ range; the region we would expect to see absorption of a red dye. The p- 
toluidine dye (6) showed a very similar trend with all three absorbances, but most notably the decrease in its absorption responsible for its green colour at $\lambda=626 \mathrm{~nm}$ (Figure $5 \mathbf{b}$ ).
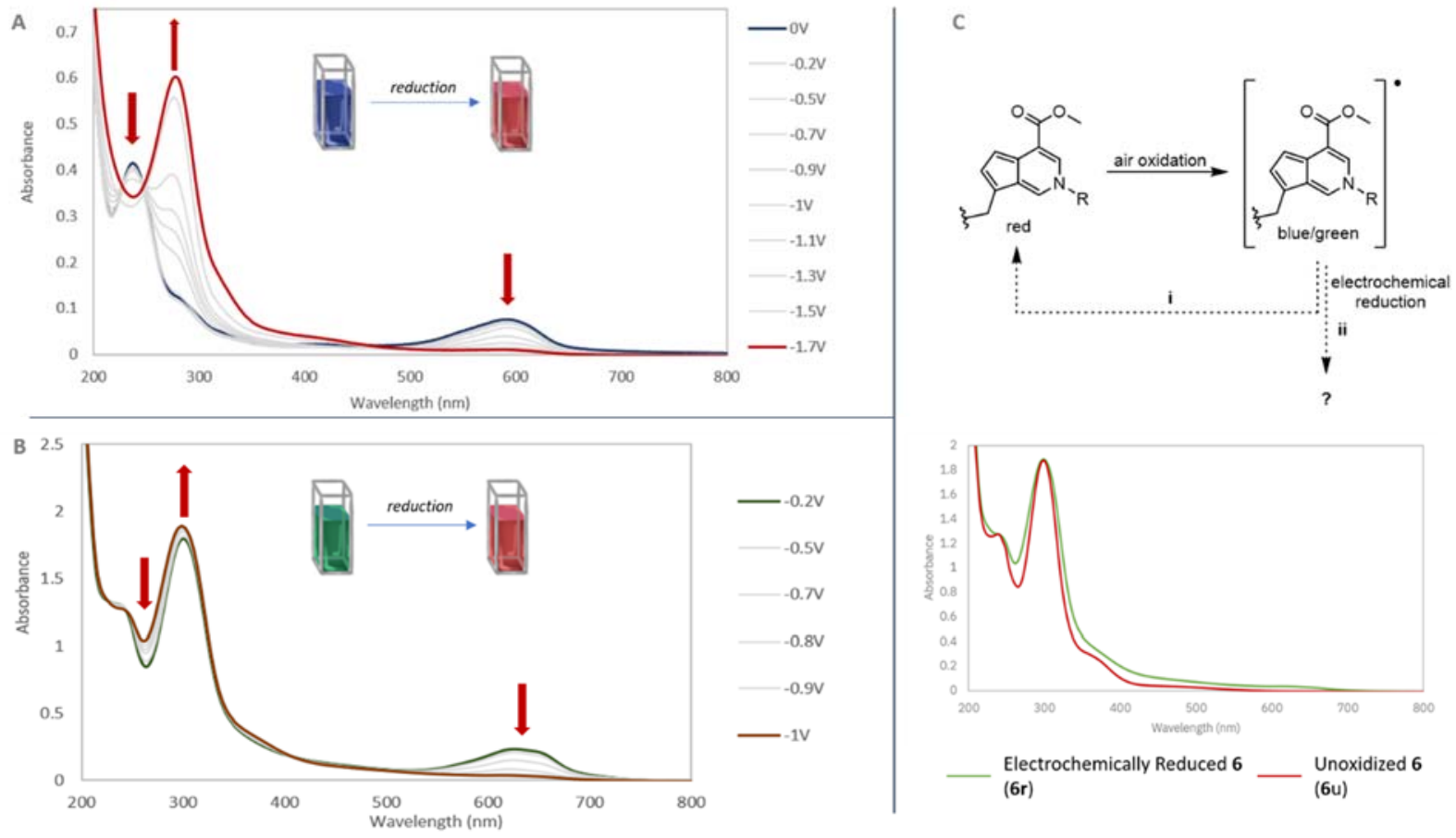

Figure 5. Spectroelectrochemistry of genipin dyes from a) methylamine (4) and b) p-toluidine (6); c) Top: proposed pathways for electrochemical reduction of the oxidized dye through pathway $\mathbf{i}$ to the parent, unoxidized dye or pathway ii to an unknown reduced species. Bottom: UV-Vis spectra of p-toluidine dyes of the reduced dye (green) and unoxidized dye (red).

The fact that both methylamine and p-toluidine dyes showed similar behavior lends credence to pathway $\boldsymbol{i}$ in Figure 5c. Nevertheless, we further directly compared the UV-VIS spectrum of the parent "unoxidized" red-dye derived from p-toluidine $(\mathbf{6 u})$ to the fully reduced spectrum generated from the spectroelectrochemistry (6r) (Figure 5c, bottom). Both spectra were nearly identical, exhibiting the same absorption features with slight broadening. This not only supports the idea that 
the electrochemical reduction proceeds through pathway $\mathbf{i}$ indicating that the reduced species and oxidized species are one and the same, but also further supports our hypothesis that the intense dye colour is the result of an open-shell configuration.

Taken together with the EPR data, these results are reminiscent of reports of emeraldine polyaniline (PANI) - the partially oxidized form of PANI. The quinoidal-radical resonance structure of this conductive polymer has been well established as an open-shell ground state for this and other conducting polymers ${ }^{[24]}$. Further, similar EPR spectral data were obtained for emeraldine PANI, supporting the theory of an open-shell configuration being the cause of the genipin dye colorations ${ }^{[25]}$. Additionally, there are parallels between these genipin-derived dyes and other $\pi$-conjugated materials and the generation of "polarons" in these materials. ${ }^{[2]}$ To highlight these similarities, Fang and co-workers recently reported the synthesis of ladder-type polyaniline analogues, resulting in materials with very similar dye spectroscopy and properties to genipin-amine dyes (reversible oxidation from red to blue materials, delocalized EPR signals, etc). ${ }^{[27,28]}$

Conclusion: In this study, we were able to show that substituting aliphatic amines for aromatic amines results in the formation of a green dye upon reaction with genipin. It was widely hypothesized that these vibrant dyes are the result of an oxidative polymerization reaction, however, analysis of the pigments made led us to revisit this hypothesis. Gel permeation chromatography and mass spectrometry revealed that no polymers are formed in either the blue or green genipin-derived dyes made with methylamine or p-toluidine, respectively. The presence of an unpaired electron was then confirmed using EPR spectroscopy, which we suggest is the source of the deeply colored genipin dyes, and not a polymerization event as previously thought. Spectroelectrochemistry analysis supported this hypothesis, showing that the UV-Vis spectrum of 
the reduced species bears a great resemblance to the red dye intermediate formed before exposure to oxygen. These results draw parallels between genipin dyes and $\pi$-conjugated materials, and these similarities may unlock a new area of research for genipin, one where it is used as a sustainable starting material for $\pi$-conjugated materials. This is an area we are actively exploring.

\section{References}

[1] A.K. Samanta, A.K. Konar, Dyeing of Textiles with Natural Dyes, Natural Dyes IntechOpen, 2011, pp. 29-56, DOI: 10.5772/21341.

[2] G. T. Sigurdson, P. Tang, M. M. Giusti, Annu. Rev. Food Sci. Technol. 2017, 8, 261-280.

[3] L. Jespersen, Lene, D. Strømdahl, K. Olsen, L. H. Skibsted, Eur. Food Res. Technol. 2005, 220, 261-266.

[4] C. A. Hobbs, M. Koyanagi, C. Swartz, J. Davis, R. Maronpot, L. Recio, S. Hayashi, Food Chem. Toxicol. 2018, 118, 695-708.

[5] I. Mallov, F. Jeeva, C. B. Caputo, J. Chem. Technol. Biotechnol. 2020, DOI: 10.1002/jctb.6556.

[6] M. F. Butler, Y.F. Ng, P. D. A. Pudney, J. Polym. Sci. Part A Polym. Chem. 2003, 41, 3941-3953.

[7] J. S. Yoo, Y. J. Kim, S. H. Kim, S. H. Choi, Korean J. Thorac. Cardiovasc. Surg. 2011, 44, 197-207.

[8] H.W. Sung, R.N. Huang, L. L. H. Huang, C.C. Tsai, C.T. Chiu, J. Biomed. Mater. Res. 1998, 42, 560-567.

[9] X. Dong, J. Liang, A. Yang, C. Wang, D. Kong, F. Lv, ACS Appl. Mater. Interfaces 2018, 10, 21861-21875.

[10] M. Robinson, S. Douglas, S. M. Willerth, Sci. Rep. 2017, 7, 6250.

[11] J. Almog, Y. Cohen, M. Azoury, T.R. Hahn, J. Forensic Sci. 2004, 49, 255-257.

[12] G. Levinton-Shamuilov, Y. Cohen, M. Azoury, A. Chaikovsky, J. Almog, J. Forensic Sci. 2005, 50, 1367-1371.

[13] B. C. Kim, H. G. Kim, S. A. Lee, S. Lim, E. H. Park, S. J. Kim, C. J. Lim, Biochem. Pharmacol. 2005, 70, 1398-1407.

[14] M. Bellefeuille, D. Peters, M. Nolin, P. Slusarewicz, D. Telgenhoff, Aust. Vet. J. 2017, 95, $167-173$.

[15] J.-E. Park, J.-Y. Lee, H.-G. Kim, T.-R. Hahn, Y.-S. Paik, J. Agric. Food Chem. 2002, 50, $6511-6514$.

[16] S. Di Tommaso, H. David, J. Gomar, F. Leroy, C. Adamo, RSC Adv. 2014, 4, 11029- 
11038.

[17] R. Touyama, Y. Takeda, K. Inoue, I. Kawamura, M. Yatsuzuka, T. Ikumoto, T. Shingu, T. Yokoi, H. Inouye, Chem. Pharm. Bull. 1994, 42, 668-673.

[18] R. Touyama, K. Inoue, Y. Takeda, M. Yatsuzuka, T. Ikumoto, N. Moritome, T. Shingu, T. Yokoi, H. Inouye, Chem. Pharm. Bull. 1994, 42, 1571-1578.

[19] S. W. Lee, J. M. Lim, S. H. Bhoo, Y. S. Paik, T. R. Hahn, Anal. Chim. Acta 2003, 480, 267-274.

[20] H. D. Nguyen, T. H. Nguyen, N. V. Hoang, N. N. Le, T. N. N. Nguyen, D. C. T. Doan, M. C. Dang, Adv. Nat. Sci. Nanosci. Nanotechnol. 2014, 5, 045001.

[21] B. G. Segal, M. Kaplan, G. K. Fraenkel, J. Chem. Phys. 1965, 43, 4191-4200.

[22] M. S. Jahan, C. Alexander, Radiat. Res. 1978, 74, 251-264.

[23] M. D. Peeks, C. E. Tait, P. Neuhaus, G. M. Fischer, M. Hoffmann, R. Haver, A. Cnossen, J. R. Harmer, C. R. Timmel, H. L. Anderson, J. Am. Chem. Soc. 2017, 139, 10461-10471.

[24] M. Canales, J. Torras, G. Fabregat, A. Meneguzzi, C. A. Alemán, J. Phys. Chem. B 2014, $118,11552-11562$.

[25] Z. D. Zujovic, M. Gizdavic-Nikolaidis, P. A. Kilmartin, J. Travas-Sejdic, R. P. Cooney, G. A. Bowmaker, Appl. Magn. Reson. 2005, 28, 123-136.

[26] J. L. Bredas, G. B. Street, Acc. Chem. Res. 1985, 18, 309-315.

[27] X. Ji, M. Leng, H. Xie, C. Wang, K. R. Dunbar, Y. Zou, L. Fang, Chem. Sci. 2020, 11, $12737-12745$.

[28] X. Ji, H. Xie, C. Zhu, Y. Zou, A. U. Mu, M. Al-Hashimi, K. R. Dunbar, L. Fang, J. Am. Chem. Soc. 2020, 142, 641-648. 